\title{
«Il ne faut pas réinventer la roue!»-Sitzung der UNION mit Bundesrat Didier Burkhalter vom 18. Februar 2011
}

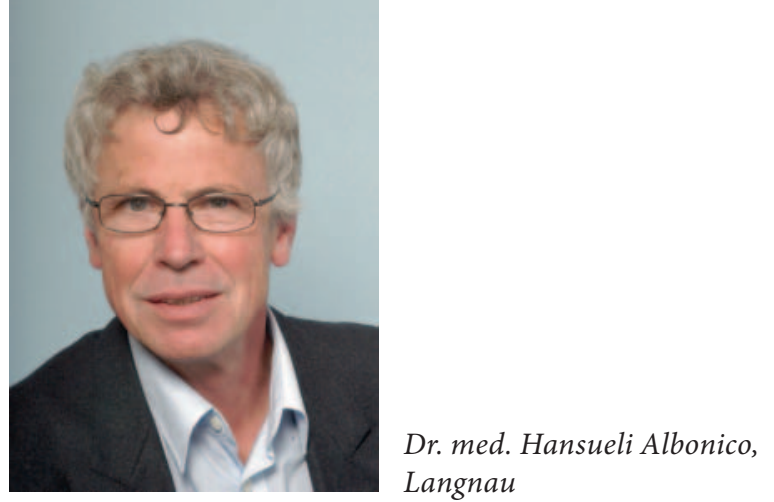

Bereits anfangs 2010 hatte die UNION Bundesrat Burkhalter um ein Treffen zur Erörterung der Besonderheiten der ärztlichen Komplementärmedizin gebeten. Dies war dazumal unter Hinweis auf das laufende Antragsverfahren abgelehnt worden. Die UNION-Fachgesellschaften hatten dann ihre neuen Anträge um Wiederaufnahme in die Grundversicherung eingereicht und entsprechend den Rückmeldungen des Bundesamts für Gesundheit (BAG) ergänzt. Die Eidgenössische Leistungs- und Grundsatzkommission (ELGK) empfahl nach zwei zusätzlichen Sitzungen am 7. Dezember die Ablehnung aller fünf Anträge und verwies dabei auf ungelöste Beurteilungsund Verfahrensfragen. So kam die Pressekonferenz von Bundesrat Burkhalter am 12. Januar 2011 überraschend: Der Gesundheitsminister beschloss entgegen der Kommissionsempfehlung die erneute Wiederaufnahme der ärztlichen Komplementärmedizin in die soziale Grundversicherung der Schweiz per 1. Januar 2012. Allerdings ist die Wiederaufnahme vorläufig bis Ende 2017 befristet und unter die Bedingung eines erneuten Nachweises der Wirksamkeit, Zweckmässigkeit und Wirtschaftlichkeit (WZW) gestellt (vergleiche Pressemitteilung Eidgenössisches Departement des Innern (EDI) vom 12.01.2011).

Die zur Konkretisierung des weiteren Vorgehens anberaumte Sitzung mit der UNION vom 18. Februar verlief vielversprechend. Teilnehmer waren Bundesrat Burkhalter mit seinem Stab, Direktor Pascal Strupler und Vizedirektor Andreas Faller vom BAG mit den Mitarbeitern Dr. Felix Gurtner und Dr. Sylvie Bailat sowie je zwei Vertreter der UNION und der fünf antragstellenden Fachgesellschaften. Der Gesundheitsminister leitete die ganze zweistündige Sitzung persönlich und sicherte auch seine weitere persönliche Mitarbeit zu; sein Führungsstil beeindruckte durch Kompetenz, Offenheit und Engagement. Burkhalter erinnerte daran, dass in der Schweiz die Verfassung über dem Gesetz und das Gesetz über den Kommissionen steht, konstatierte jedoch: «Nicht nur unsere Medizin, die ganze Schweiz hat eine Vertrauenskrise.» Diese gelte es in allen Bereichen zu überwinden. Burkhalters Bekenntnis zu Dialog und Vertrauensbildung vermochte zu überzeugen.

Namens der UNION fasste der Präsident Hansueli Albonico deren Position nochmals zusammen: Die UNION hat sich stets zum WZW-Nachweis bekannt, in der Meinung, dass dieser grundsätzlich auch für die ärztliche Komplementärmedizin zu erbringen sei. Die UNION sei dankbar, dass Bundesrat Burkhalter dem Problem des adäquaten Wirksamkeitsnachweises die angemessene Aufmerksamkeit schenke. Medizin, auch die sog. Schulmedizin, sei nie reine Naturwissenschaft. Und Evidence-Based Medicine beschränke sich nicht auf randomisierte kontrollierte Studien. Die Doppelblindstudie sei nicht das geeignete Instrument zur Beurteilung ganzer Fachrichtungen; jede Art der Hausarztmedizin brauche praxisnahe Real-World-Forschungsdesigns.

\section{Agenda UNION}

\section{Vorstandssitzung UNION}

Delegiertenversammlung UNION 2011

Vorstandssitzung UNION

Vorstandssitzung UNION
Donnerstag, 5. Mai 2011 (Vormittag); Bern

Donnerstag, 5. Mai 2011 (Nachmittag); Bern

Donnerstag, 30. Juni 2011 (Nachmittag); Bern

Donnerstag, 1. September 2011 (Nachmittag); Bern 
Die UNION begrüsst deshalb das von Bundesrat Burkhalter anlässlich seiner Pressekonferenz vom 12. Januar 2011 vorgeschlagene Prozedere, nämlich:

1. Identifizierung der verbleibenden umstrittenen Aspekte im Antrag jeder einzelnen Fachgesellschaft.

2. Erarbeitung eines verlässlichen Evaluationskonzepts mit gezielter Operationalisierung der HTA (Health Technology Assessment)-Vorgaben.

3. Gezielte Ergänzung der Anträge durch die Fachgesellschaften mit Evaluation durch eine international anerkannte Institution.

4. Revision der ELGK.

Die UNION begrüsst des Weiteren, dass das EDI in $\mathrm{Zu}$ sammenarbeit mit den betroffenen Kreisen alle notwendigen Massnahmen zur Konkretisierung der weiteren Anliegen des neuen Verfassungsartikels 118a ergreifen will, speziell hinsichtlich einer Erleichterung der Zulassung von komplementärmedizinischen und traditionellen Arzneimitteln, der Förderung der Forschung sowie der Schaffung von Instituten oder Lehrstühlen für Komplementärmedizin an unseren Universitäten.

Zur Implementierung des neuen WiederaufnahmeProzederes sind die Fachgesellschaften angehalten, selber bis Ende 2011 ein Evaluationskonzept vorzulegen, das auch politisch abgestützt sein sollte. Gemäss diesem Konzept müssen die Anträge bis 2015 ergänzt werden. Vorab sollen in Zusammenarbeit mit dem BAG die von der ELGK angemahnten Lücken in den fünf Anträgen identifiziert und bewertet werden. Gleichzeitig wird das EDI seinerseits eine international anerkannte HTA-Institution mit einer unabhängigen Evaluation beauftragen. Zudem sollen Zusammensetzung und Mandatierung der ELGK durch das EDI revidiert werden.
Schliesslich setzt das EDI in Absprache mit Dakomed und UNION eine Begleitgruppe ein, die für die übergeordnete Begleitung des gesamten Konkretisierungsprozesses des Verfassungsartikels, also auch für die Belange der nichtärztlichen Komplementärtherapie und Alternativmedizin sowie der Förderung der Heilmittel, zuständig sein soll. Mit der Leitung hat das EDI Frau Semya Ayoubi betraut.

«Il ne faut pas réinventer la roue» - diese Aussage von Bundesrat Burkhalter anlässlich der Sitzung sollte für alle Player im neuen Antragsprozess richtungsweisend sein. Die UNION-Fachgesellschaften haben in den bisherigen Anträgen 2005 und 2010 bereits gute WZW-Evidenz aufgearbeitet - diese gilt es zu einer sachgemässen, kompetenten Würdigung zu bringen. Echte und vermeintliche Lücken gilt es zu identifizieren und mit vernünftigem Aufwand zu schliessen. Die vom EDI eingesetzte HTAInstitution muss die Anträge unter verlässlicher Festlegung fairer Evaluationskriterien neu beurteilen. Die übergeordnete Begleitgruppe schliesslich muss die Korrektheit und Fairness des gesamten Prozesses sicherstellen.

«Eigentlich waren die Komplementärmediziner zu früh», meinte Bundesrat Burkhalter abschliessend unter Würdigung der Erkenntnis, dass adäquate Instrumente zur Beurteilung ganzer Fachrichtungen bisher eigentlich gar nicht bereitstanden. Er ist zuversichtlich, dass sein dezidiertes Vorgehen bis spätestens 2017 zu einer befriedigenden Lösung führen wird, die eine Gesetzesänderung unnötig macht.

20. Februar 2011

Dr. med. Hansueli Albonico Präsident Union schweizerischer komplementärmedizinischer Ärzteorganisationen 